\title{
Algal cover and sea urchin spatial distribution at Madeira Island (NE Atlantic)*
}

\author{
FILIPE M. A. ALVES ${ }^{1}$, LUÍS M. CHÍCHARO ${ }^{1}$, ESTER SERRÃO ${ }^{1}$ \\ and ANTÓNIO D. ABREU² \\ ${ }^{1}$ Centro de Ciências do Mar, Universidade do Algarve, Faculdade de Ciências do Mar e do Ambiente, Campus de \\ Gambelas, 8000-810 Faro, Portugal. E-mail: filalves@ rocketmail.com \\ ${ }^{2}$ Estação de Biologia Marinha do Funchal, Cais do Carvão, Promenade da Orla Marítima do Funchal, Gorgulho, \\ 9000-107 Funchal, Portugal.
}

\begin{abstract}
SUMMARY: This study describes sea urchin spatial distribution in relation to environmental factors, and the relationship between Diadema antillarum density and algal abundance. Twenty-three transects around Madeira Island were surveyed by scuba divers, and sea urchin density and algal cover were determined in situ. Sampling sites along these transects were characterised in terms of distance from the tide line, water depth, substratum type, bottom declivity and water turbulence. Diadema antillarum was the dominant sea urchin species. Paracentrotus lividus and Arbacia lixula occurred at shallower depths (2-6 m), contrasting with the distribution of Sphaerechinus granularis, which occurs among D. antillarum (4-20 m). Surveys found two alternative types of communities on rocky shores: 1) a community with high algal cover and low numbers of sea urchins, along the north and south-west coasts and; 2) a community with little algal cover and high densities of sea urchins, along the south-east coast. Macroalgal cover and D. antillarum densities were inversely correlated (adjusted $\mathrm{R}^{2}=75.6 \% ; \mathrm{n}=429 ; \mathrm{p}<0.05$ ). The results showed that water turbulence was the most important factor limiting the distribution of $D$. antillarum on rocky substrates. We propose a multiple non-linear regression model (using backward stepwise analysis) to explain $D$. antillarum abundance on the rocky shores: $D$. antillarum $/ \mathrm{m}^{2}(\sqrt{ })=0.121-0.209$ distance from shore (in m) $(\sqrt{ } \sqrt{ })+2.052$ water depth (in m) $(\sqrt{ } \sqrt{ })-1.778$ water turbulence level $(\sqrt{ } \sqrt{ })-0.007$ water turbulence level ${ }^{4}(\sqrt{ } \sqrt{ })$; where $\sqrt{ } \sqrt{ }$ indicates data are square-root transformed (adjusted $\mathrm{R}^{2}=60.99 \% ; \mathrm{n}=454 ; \mathrm{p}<0.05$ ).
\end{abstract}

Key words: sea urchins, Madeira Island, algae, Diadema antillarum, distribution.

\section{INTRODUCTION}

Several studies of sea urchin distribution and the effects of this on algal populations have been reported world-wide (e.g. Valdez and Villalobos, 1978; Bauer, 1980; Weil et al., 1984; Benedetti-Cecchi and Cinelli, 1995; Turon et al., 1995; Falcon et al., 1996). Sea urchins are known to control the abundance and distribution of algae and can therefore have a profound influence on the structure of benth-

\footnotetext{
*Received August 31, 2000. Accepted March 22, 2001.
}

ic communities (Andrew, 1989; Vadas, 1990; Vadas and Elner, 1992; Valentine et al., 1997). At Madeira Island (Portugal, north-east Atlantic), sea urchin ecology has not previously been studied in detail, although it is referred to by Augier (1985), Wirtz (1995, 1998) and Bianchi et al. (1998).

Sea urchin species known to occur on the rocky shores of Madeira are Paracentrotus lividus (Lamarck), Arbacia lixula (Lamarck), Sphaerechinus granularis (Lamarck), Diadema antillarum (Philippi), and the less common Arbaciella elegans (Mortensen) and Centrostephanus longispi- 
nus (Philippi) (Wirtz, 1995). Paracentrotus lividus and A. lixula have been studied in the Mediterranean Sea, where they play a decisive role in the development of benthic macrophyte communities (Kempf, 1962; Bergin, 1987; Benedetti-Cecchi and Cinelli, 1995; Turon et al., 1995; Fernandez and Boudouresque, 1997). The distribution of $S$. granularis has previously been studied only at Faial (Azores), by Maciel and Gonçalves (1998).

At Madeira, empirical observations suggest that $D$. antillarum abundance is increasing (Abreu et al., 1995; personal observations), which seems to be reflected by reduced seaweed abundance. This sea urchin species, and its ability to affect ecosystems, has not yet been studied around Madeira, though it has been widely studied in the western Atlantic, where it has a great impact on benthic community structure (e.g. Sammarco et al., 1974; Sammarco, 1980, 1982a, b; Carpenter, 1981, 1984; Liddel and Ohlhorst, 1986, 1992; Lessios, 1988a, b, 1995). Several studies have found that the spatial distribution of $D$. antillarum correlates with a number of other factors, such as the substratum type (Valdez and Villalobos, 1978), structural morphology of the reef, food availability, predation pressure (Weil et al., 1984), aggressive territorial behaviour of fishes (Sammarco and Williams, 1982), and water turbulence (Casañas et al., 1998). However, an integrated analysis of the major factors has not previously been undertaken. The aims of this study are to describe algal cover and sea urchin distribution around Madeira, and to investigate the importance of several environmental factors in determining $D$. antillarum abundance.

\section{STUDY SITE}

Madeira Island is located in the northeast Atlantic Ocean, at approximately $32^{\circ} 38^{\prime} \mathrm{N}$, 1654'W (Fig. 1; Biscoito and Abreu, 1998). The island, of volcanic origin, is the largest of the archipelago bearing the same name, which includes Porto Santo and the smaller Desertas and Selvagens islands. Madeira extends for $58 \mathrm{~km}$ along a WNW to ESE axis, and its $153 \mathrm{~km}$ of coastline is predominantly rocky. Sea surface temperatures usually range between 17 and $22.5^{\circ} \mathrm{C}$. Typically, the presence of a subtropical anticyclone over the Azores causes a northeasterly wind and ocean current, with an average velocity of 0.5 knots, to persist for most of the year at the islands (Abreu and Biscoito, 1998). The Madeira archipelago is an important biogeographical region because it contains a large number of endemic species (Baez, 1993; Beyhl et al., 1995; Stock, 1995).

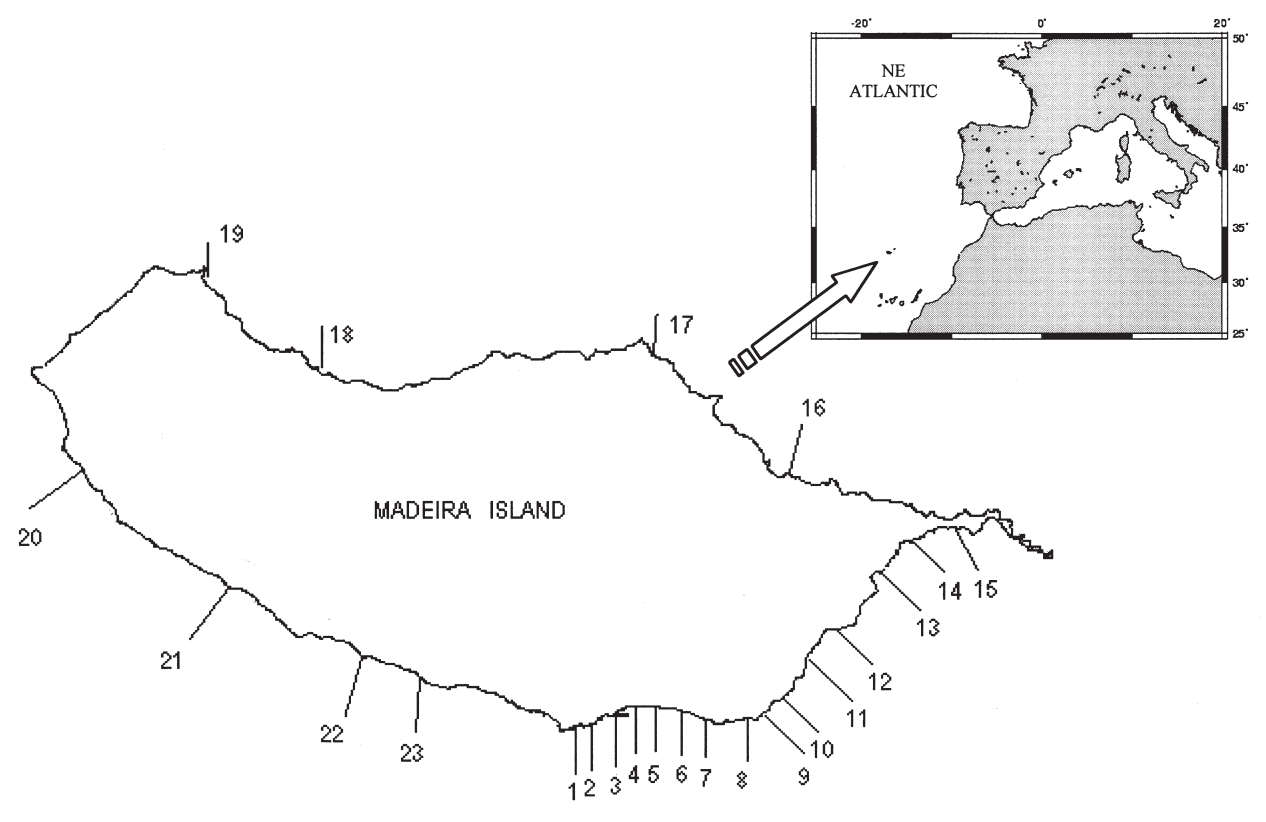

FIG. 1. - Location of transects along the Madeira coast: $\mathbf{1}$ - Clube Naval (west); $\mathbf{2}$ - Lido (west); $\mathbf{3}$ - Molhe; 4 - Barreirinha (east); 5 - Lazareto; 6 - Pináculo; 7 - Garajau; 8 - Galo; 9 - Roca-mar; 10 - Reis Magos (east); 11 - Porto Novo; 12 - Santa Cruz (east quay); 13 - Machico (quay); 14 - Caniçal (Junta quay); 15 - Quinta do Lorde; 16 - Porto da Cruz (quay); 17 - São Jorge; 18 - Seixal (quay); 19 - Porto Moniz (quay); 20 - Paúl do Mar (west); 21 - Madalena (quay); 22 - Ribeira Brava (east harbour); 23 - Campanário (islet). 


\section{METHODOLOGY}

Off Madeira Island, 23 transects were surveyed by scuba divers from July to August 1998: transects 1 to 15 were off the southeast coast (1SE-15SE); transects 16 to 19 were off the north coast $(16 \mathrm{~N}-19 \mathrm{~N})$, and transects 20 to 23 were off the southwest coast (20SW-23SW) (Fig. 1). Along each transect, the number of sea urchins per square metre, the percentage of algal cover, water depth, substratum type and bottom declivity were assessed and recorded on waterproof sheets every $10 \mathrm{~m}$, starting from about 2 $\mathrm{m}$ distance from shore and continuing until sandy sediments (where sea urchins are scarce) occurred. When weather conditions were adverse, data collection began from $10 \mathrm{~m}$ distance from shore. Every 10 $\mathrm{m}$ along each transect, three random replicates of the number of sea urchins and of the percentage of algal cover were assessed within a $1 \times 1 \mathrm{~m}$ quadrat and a $0.5 \times 0.5 \mathrm{~m}$ quadrat divided into $1005 \times 5 \mathrm{~cm}$ squares respectively. At each sampling point, all sea urchins were identified and counted.

Six different categories of substratum were recorded: rock; big boulders; big and medium boulders; medium and small boulders; small boulders; and sand. We considered big boulders to be larger than $1 \mathrm{~m}$, medium boulders between 0.2 and $1 \mathrm{~m}$, and small boulders smaller than $0.2 \mathrm{~m}$. Bottom declivity was measured to four levels: 1) $0-5^{\circ}$; 2) $6-30^{\circ}$; 3) $31-60^{\circ}$; 4) $61-90^{\circ}$. The degree of water turbulence at each transect was categorised from annual wave-height frequency $(\%)$ data recorded by the Portuguese Hydrographic Institute between 1961 and 1970 off the major coastal areas of Madeira (Instituto Hidrográfico, 1979). Based on these data, four categories were recognised: 1) total calm or very low disturbance, $99-100 \%$ frequency of waves less than $1.5 \mathrm{~m}$ in height; 2) moderate disturbance, $98 \%$ of waves less than $1.5 \mathrm{~m}$ and $2 \%$ from $2-3.5 \mathrm{~m}$ in height; 3 ) considerable disturbance, $77 \%$ less than $1.5 \mathrm{~m}$ and $23 \%$ from $2-3.5 \mathrm{~m}$ in height; 4) strong or very strong disturbance, $54 \%$ less than $1.5 \mathrm{~m}$ and $46 \%$ from $2-3.5 \mathrm{~m}$.

In an attempt to determine the factors limiting the spatial distribution of $D$. antillarum on rocky shores (e.g. distance from shore, water depth, substratum type, bottom declivity and water turbulence), a multiple non-linear regression (with corresponding ANOVA) was performed. Variables used for the equation were selected based on a backward stepwise analysis using the Statistica V.5 program. Data were square-root transformed since this improved the residuals.
To test for differences in sea urchin distributions, a two-way ANOVA test was performed. Water depth and sea urchin species were the independent variables, and sea urchin density was the dependent variable.

A linear regression between the percentage of algal cover and $D$. antillarum density was performed using the Minitab 12.2 statistics program. Variables were square-root transformed as this minimised the residuals.

\section{RESULTS}

\section{Species distribution and abundance}

Four sea urchin species were found during the course of the surveys: Diadema antillarum, Arbacia lixula, Sphaerechinus granularis and Paracentrotus lividus. Diadema antillarum was found in high densities off the southeast coast on transects 1SE to 15SE, and also on transects 23SW (on the border of the southeast coast) and 19N (Tables 1 and 2). Mean density in these areas was $6 \pm 4.8$ (standard deviation) individuals $/ \mathrm{m}^{2}$. We only found individuals with a test diameter greater than $10 \mathrm{~mm}$. Between transects 23SW to 1SE (Praia Formosa) and 14SE to 15SE (Prainha), the substratum was sandy and sea urchins were almost completely absent. On the north and southwest coasts of the island, with the exception of transect $19 \mathrm{~N}$, very low densities of sea urchins, particularly $D$. antillarum, were observed. In these areas, A. lixula was the predominant sea urchin, although it occurred in very low densities (between 0 and 1 individuals $/ \mathrm{m}^{2}$ ). However, on the south coast, mean densities of $17 \pm 1.5,14 \pm 16.5,8 \pm 1.2$ and $6 \pm 4.5$ individuals $/ \mathrm{m}^{2}$ were observed. Sphaerechinus granularis was generally observed at densities lower than $2 \pm 0.6$ individuals $/ \mathrm{m}^{2}$. Paracentrotus lividus was only recorded on transect $1 \mathrm{SE}$, at $1.8 \mathrm{~m}$ depth, with a mean density of $11 \pm 1.5$ individuals $/ \mathrm{m}^{2}$ and on transect $5 \mathrm{SE}$, at $3 \mathrm{~m}$ depth, at a mean density of $2 \pm 4.0$ individuals $/ \mathrm{m}^{2}$ (Tables 1 and 2 ).

\section{Distance from tide line and water depth}

Sea urchin species distribution varied with distance from tide line and water depth. Diadema antillarum was the dominant species at almost all distances from the shore and at all depths, except in areas shallower than 2-3 m. However, on transect 1SE, a maximum of 68 individuals $/ \mathrm{m}^{2}$ of $D$. antillarum was recorded at $4 \mathrm{~m}$ depth, and in the Porto Santo Island 
TABLE 1. - Sea urchin density and percent algal cover (mean of three samples \pm standard deviation), water depth, bottom declivity (Bott. decliv.) and substrate type against distance (Dist.) from the tide line for transects 1 to 13 . (Measurements were taken every $10 \mathrm{~m}$ until sandy sediments were reached, with the exception of those marked with an asterisk (*). Bottom declivity was measured on a four-level scale as described in the methodology.)

\begin{tabular}{|c|c|c|c|c|c|c|c|c|c|}
\hline Site & $\begin{array}{c}\text { Dist. from } \\
\text { shore }(\mathrm{m})\end{array}$ & $\begin{array}{r}\text { Sea urcl } \\
\text { D. antillarum }\end{array}$ & $\begin{array}{l}\mathrm{s} / \mathrm{m}^{2} \text { (mean } \\
\text { A. lixula }\end{array}$ & $\begin{array}{l}\text { standard deviation) } \\
\text { S. granularis }\end{array}$ & P. lividus & $\begin{array}{c}\text { Algal } \\
\text { cover }(\%)\end{array}$ & $\begin{array}{l}\text { Depth } \\
(\mathrm{m})\end{array}$ & $\begin{array}{l}\text { Bott. } \\
\text { decliv. }\end{array}$ & $\begin{array}{l}\text { Substratum } \\
\text { type }\end{array}$ \\
\hline \multirow[t]{5}{*}{1} & 2 & $0.0 \pm 0.0$ & $17.3 \pm 1.5$ & $0.0 \pm 0.0$ & $10.7 \pm 1.5$ & $68.3 \pm 17.6$ & 1.8 & 1 & rock \\
\hline & 10 & $50.7 \pm 18.6$ & $0.0 \pm 0.0$ & $0.0 \pm 0.0$ & $0.0 \pm 0.0$ & $14.3 \pm 2.1$ & 3.8 & 3 & rock \\
\hline & 20 & $21.0 \pm 5.6$ & $0.0 \pm 0.0$ & $0.0 \pm 0.0$ & $0.0 \pm 0.0$ & $10.3 \pm 13.1$ & 6 & 1 & rock \\
\hline & 30 & $15.7 \pm 2.1$ & $0.0 \pm 0.0$ & $0.0 \pm 0.0$ & $0.0 \pm 0.0$ & $20.3 \pm 17.6$ & 6.5 & 3 & rock \\
\hline & 40 & $11.3 \pm 3.1$ & $0.0 \pm 0.0$ & $0.0 \pm 0.0$ & $0.0 \pm 0.0$ & $0.0 \pm 0.0$ & 10 & 2 & big boulders \\
\hline \multirow[t]{8}{*}{2} & 2 & $0.0 \pm 0.0$ & $1.0 \pm 1.0$ & $0.0 \pm 0.0$ & $0.0 \pm 0.0$ & $86.0 \pm 14.4$ & 1.5 & 2 & big and med. bld. \\
\hline & 10 & $0.0 \pm 0.0$ & $0.0 \pm 0.0$ & $1.3 \pm 0.6$ & $0.0 \pm 0.0$ & $96.3 \pm 2.5$ & 4 & 2 & big and med. bld. \\
\hline & 20 & $4.0 \pm 1.0$ & $0.7 \pm 1.2$ & $0.3 \pm 0.6$ & $0.0 \pm 0.0$ & $87.3 \pm 10.7$ & 5.5 & 2 & big and med. bld. \\
\hline & 30 & $2.3 \pm 2.1$ & $0.0 \pm 0.0$ & $2.0 \pm 0.0$ & $0.0 \pm 0.0$ & $5.7 \pm 2.1$ & 6.5 & 2 & big and med. bld. \\
\hline & 40 & $5.0 \pm 1.0$ & $0.0 \pm 0.0$ & $0.7 \pm 0.6$ & $0.0 \pm 0.0$ & $8.3 \pm 4.7$ & 9 & 3 & big and med. bld. \\
\hline & 50 & $5.3 \pm 0.6$ & $0.0 \pm 0.0$ & $0.0 \pm 0.0$ & $0.0 \pm 0.0$ & $7.0 \pm 2.0$ & 10 & 2 & big and med. bld. \\
\hline & 60 & $7.3 \pm 0.6$ & $0.0 \pm 0.0$ & $0.0 \pm 0.0$ & $0.0 \pm 0.0$ & $8.3 \pm 2.9$ & 12 & 2 & big and med. bld. \\
\hline & 70 & $5.7 \pm 2.1$ & $0.0 \pm 0.0$ & $0.0 \pm 0.0$ & $0.0 \pm 0.0$ & $5.3 \pm 3.2$ & 12.9 & 2 & big and med. bld. \\
\hline \multirow[t]{3}{*}{3} & 2 & $5.3 \pm 2.3$ & $5.7 \pm 4.5$ & $0.0 \pm 0.0$ & $0.0 \pm 0.0$ & $3.3 \pm 2.5$ & 5 & 3 & big boulders \\
\hline & 10 & $14.0 \pm 2.0$ & $0.7 \pm 1.2$ & $0.0 \pm 0.0$ & $0.0 \pm 0.0$ & $3.7 \pm 1.5$ & 9 & 3 & big boulders \\
\hline & 20 & $8.0 \pm 4.0$ & $0.0 \pm 0.0$ & $0.0 \pm 0.0$ & $0.0 \pm 0.0$ & $2.3 \pm 0.6$ & 19 & 3 & big boulders \\
\hline \multirow[t]{6}{*}{4} & 2 & $0.0 \pm 0.0$ & $0.3 \pm 0.6$ & $0.0 \pm 0.0$ & $0.0 \pm 0.0$ & $98.0 \pm 1.0$ & 3.5 & 2 & big and med. bld. \\
\hline & 10 & $0.0 \pm 0.0$ & $0.0 \pm 0.0$ & $0.7 \pm 1.2$ & $0.0 \pm 0.0$ & $75.0 \pm 9.6$ & 5.7 & 2 & med.andsmall bld. \\
\hline & 20 & $0.0 \pm 0.0$ & $0.0 \pm 0.0$ & $6.7 \pm 1.2$ & $0.0 \pm 0.0$ & $4.3 \pm 1.5$ & 6 & 2 & small boulders \\
\hline & 30 & $0.3 \pm 0.6$ & $0.0 \pm 0.0$ & $3.3 \pm 3.1$ & $0.0 \pm 0.0$ & $7.3 \pm 1.5$ & 7.5 & 2 & small boulders \\
\hline & 40 & $1.0 \pm 1.0$ & $0.3 \pm 0.6$ & $1.0 \pm 1.0$ & $0.0 \pm 0.0$ & $86.3 \pm 8.1$ & 9 & 2 & big and med. bld. \\
\hline & 50 & $1.0 \pm 1.0$ & $0.0 \pm 0.0$ & $0.7 \pm 1.2$ & $0.0 \pm 0.0$ & $90.7 \pm 1.2$ & 10 & 2 & big and med. bld. \\
\hline \multirow[t]{6}{*}{5} & 2 & $0.0 \pm 0.0$ & $0.0 \pm 0.0$ & $0.0 \pm 0.0$ & $0.0 \pm 0.0$ & $84.7 \pm 9.0$ & 1.5 & 2 & big boulders \\
\hline & 10 & $0.0 \pm 0.0$ & $0.3 \pm 0.6$ & $0.0 \pm 0.0$ & $2.3 \pm 4.0$ & $91.3 \pm 3.1$ & 3 & 2 & big boulders \\
\hline & 20 & $2.7 \pm 0.6$ & $0.0 \pm 0.0$ & $0.7 \pm 1.2$ & $0.0 \pm 0.0$ & $14.0 \pm 15.6$ & 4 & 2 & big boulders \\
\hline & 30 & $4.0 \pm 1.0$ & $0.0 \pm 0.0$ & $2.3 \pm 0.6$ & $0.0 \pm 0.0$ & $12.3 \pm 5.1$ & 5 & 2 & big boulders \\
\hline & 40 & $4.3 \pm 1.5$ & $0.3 \pm 0.6$ & $0.3 \pm 0.6$ & $0.0 \pm 0.0$ & $17.0 \pm 7.8$ & 8 & 2 & big boulders \\
\hline & 50 & $2.3 \pm 2.5$ & $0.0 \pm 0.0$ & $0.7 \pm 1.2$ & $0.0 \pm 0.0$ & $10.7 \pm 2.3$ & 12 & 3 & big boulders \\
\hline \multirow[t]{9}{*}{6} & 2 & $0.0 \pm 0.0$ & $0.0 \pm 0.0$ & $0.0 \pm 0.0$ & $0.0 \pm 0.0$ & $78.0 \pm 3.0$ & 2 & 2 & big boulders \\
\hline & 10 & $0.0 \pm 0.0$ & $0.0 \pm 0.0$ & $0.0 \pm 0.0$ & $0.0 \pm 0.0$ & $63.0 \pm 14.4$ & 3.9 & 2 & big boulders \\
\hline & 20 & $3.0 \pm 1.0$ & $1.0 \pm 1.7$ & $0.0 \pm 0.0$ & $0.0 \pm 0.0$ & $3.0 \pm 3.5$ & 4.9 & 2 & big and med. bld. \\
\hline & 30 & $5.7 \pm 2.1$ & $0.3 \pm 0.6$ & $0.0 \pm 0.0$ & $0.0 \pm 0.0$ & $6.3 \pm 5.9$ & 5.5 & 2 & big and med. bld. \\
\hline & 40 & $3.7 \pm 1.5$ & $0.0 \pm 0.0$ & $0.0 \pm 0.0$ & $0.0 \pm 0.0$ & $4.3 \pm 2.1$ & 6.5 & 2 & big and med. bld. \\
\hline & 50 & $4.7 \pm 1.5$ & $0.0 \pm 0.0$ & $0.0 \pm 0.0$ & $0.0 \pm 0.0$ & $1.7 \pm 1.2$ & 9 & 3 & big and med. bld. \\
\hline & 60 & $2.7 \pm 1.2$ & $0.0 \pm 0.0$ & $0.0 \pm 0.0$ & $0.0 \pm 0.0$ & $3.3 \pm 1.5$ & 11.1 & 2 & big and med. bld. \\
\hline & 70 & $5.0 \pm 1.7$ & $0.0 \pm 0.0$ & $0.3 \pm 0.6$ & $0.0 \pm 0.0$ & $3.7 \pm 0.6$ & 14.3 & 3 & big and med. bld. \\
\hline & 80 & $2.7 \pm 1.5$ & $0.0 \pm 0.0$ & $0.0 \pm 0.0$ & $0.0 \pm 0.0$ & $2.7 \pm 0.6$ & 19.3 & 3 & big and med. bld. \\
\hline \multirow[t]{7}{*}{7} & 10 & $0.0 \pm 0.0$ & $0.0 \pm 0.0$ & $0.0 \pm 0.0$ & $0.0 \pm 0.0$ & $66.0 \pm 3.6$ & 2 & 2 & small boulders \\
\hline & 20 & $0.0 \pm 0.0$ & $0.0 \pm 0.0$ & $0.0 \pm 0.0$ & $0.0 \pm 0.0$ & $65.7 \pm 4.7$ & 5.6 & 3 & small boulders \\
\hline & 30 & $0.0 \pm 0.0$ & $0.0 \pm 0.0$ & $0.0 \pm 0.0$ & $0.0 \pm 0.0$ & $88.0 \pm 3.6$ & 7.1 & 2 & big boulders \\
\hline & 40 & $5.7 \pm 1.5$ & $0.0 \pm 0.0$ & $0.0 \pm 0.0$ & $0.0 \pm 0.0$ & $2.0 \pm 1.7$ & 8.9 & 2 & big boulders \\
\hline & 50 & $9.3 \pm 2.5$ & $0.0 \pm 0.0$ & $0.0 \pm 0.0$ & $0.0 \pm 0.0$ & $4.0 \pm 5.2$ & 9 & 2 & big boulders \\
\hline & 60 & $6.7 \pm 0.6$ & $0.0 \pm 0.0$ & $0.0 \pm 0.0$ & $0.0 \pm 0.0$ & $5.7 \pm 2.5$ & 10.5 & 3 & big boulders \\
\hline & $70 *$ & $8.3 \pm 0.6$ & $0.0 \pm 0.0$ & $0.0 \pm 0.0$ & $0.0 \pm 0.0$ & $7.7 \pm 4.2$ & 11.8 & 3 & big boulders \\
\hline \multirow[t]{8}{*}{8} & 2 & $0.0 \pm 0.0$ & $0.0 \pm 0.0$ & $0.0 \pm 0.0$ & $0.0 \pm 0.0$ & $81.0 \pm 3.5$ & 5 & 2 & med.andsmall bld. \\
\hline & 10 & $8.0 \pm 3.5$ & $0.0 \pm 0.0$ & $0.0 \pm 0.0$ & $0.0 \pm 0.0$ & $33.7 \pm 3.2$ & 5.6 & 2 & med.andsmall bld. \\
\hline & 20 & $0.0 \pm 0.0$ & $0.0 \pm 0.0$ & $0.0 \pm 0.0$ & $0.0 \pm 0.0$ & $60.3 \pm 12.9$ & 6.3 & 2 & med.andsmall bld. \\
\hline & 30 & $11.7 \pm 2.1$ & $0.0 \pm 0.0$ & $0.0 \pm 0.0$ & $0.0 \pm 0.0$ & $0.0 \pm 0.0$ & 9.9 & 3 & big and med. bld. \\
\hline & 40 & $12.3 \pm 2.1$ & $0.0 \pm 0.0$ & $0.0 \pm 0.0$ & $0.0 \pm 0.0$ & $0.0 \pm 0.0$ & 13 & 3 & big and med. bld. \\
\hline & 50 & $6.0 \pm 1.0$ & $0.0 \pm 0.0$ & $0.0 \pm 0.0$ & $0.0 \pm 0.0$ & $0.0 \pm 0.0$ & 16 & 3 & big and med. bld. \\
\hline & 60 & $7.7 \pm 2.1$ & $0.0 \pm 0.0$ & $0.0 \pm 0.0$ & $0.0 \pm 0.0$ & $0.0 \pm 0.0$ & 18.5 & 3 & big boulders \\
\hline & 70 & $8.0 \pm 5.6$ & $0.0 \pm 0.0$ & $0.0 \pm 0.0$ & $0.0 \pm 0.0$ & $0.0 \pm 0.0$ & 20.5 & 3 & big boulders \\
\hline \multirow[t]{7}{*}{9} & 2 & $0.0 \pm 0.0$ & $0.0 \pm 0.0$ & $0.0 \pm 0.0$ & $0.0 \pm 0.0$ & $0.0 \pm 0.0$ & 4.7 & 2 & big and med. bld. \\
\hline & 10 & $6.3 \pm 0.6$ & $0.0 \pm 0.0$ & $0.0 \pm 0.0$ & $0.0 \pm 0.0$ & $0.0 \pm 0.0$ & 6.8 & 2 & big and med. bld. \\
\hline & 20 & $9.3 \pm 0.6$ & $0.0 \pm 0.0$ & $0.0 \pm 0.0$ & $0.0 \pm 0.0$ & $0.0 \pm 0.0$ & 9.9 & 2 & big and med. bld. \\
\hline & 30 & $15.3 \pm 4.2$ & $0.0 \pm 0.0$ & $0.0 \pm 0.0$ & $0.0 \pm 0.0$ & $0.0 \pm 0.0$ & 11.8 & 4 & big boulders \\
\hline & 40 & $7.3 \pm 4.2$ & $0.0 \pm 0.0$ & $0.0 \pm 0.0$ & $0.0 \pm 0.0$ & $0.0 \pm 0.0$ & 15.6 & 2 & big boulders \\
\hline & 50 & $6.3 \pm 3.2$ & $0.0 \pm 0.0$ & $0.0 \pm 0.0$ & $0.0 \pm 0.0$ & $0.0 \pm 0.0$ & 16.6 & 3 & rock \\
\hline & 60 & $4.3 \pm 3.2$ & $0.0 \pm 0.0$ & $0.0 \pm 0.0$ & $0.0 \pm 0.0$ & $0.0 \pm 0.0$ & 20.1 & 4 & rock \\
\hline \multirow[t]{7}{*}{10} & 2 & $10.0 \pm 4.4$ & $0.0 \pm 0.0$ & $0.0 \pm 0.0$ & $0.0 \pm 0.0$ & $15.7 \pm 2.1$ & 5.2 & 2 & med.andsmall bld. \\
\hline & 10 & $7.0 \pm 1.7$ & $0.0 \pm 0.0$ & $0.0 \pm 0.0$ & $0.0 \pm 0.0$ & $1.3 \pm 0.6$ & 6.6 & 2 & small boulders \\
\hline & 20 & $8.0 \pm 2.0$ & $0.0 \pm 0.0$ & $0.0 \pm 0.0$ & $0.0 \pm 0.0$ & $5.7 \pm 3.1$ & 7.7 & 2 & small boulders \\
\hline & 30 & $6.0 \pm 3.6$ & $0.0 \pm 0.0$ & $1.3 \pm 1.5$ & $0.0 \pm 0.0$ & $1.7 \pm 1.2$ & 9.2 & 2 & small boulders \\
\hline & 40 & $6.3 \pm 2.9$ & $0.3 \pm 0.6$ & $2.3 \pm 0.6$ & $0.0 \pm 0.0$ & $1.0 \pm 0.0$ & 10.8 & 2 & small boulders \\
\hline & 50 & $8.3 \pm 1.2$ & $0.0 \pm 0.0$ & $0.0 \pm 0.0$ & $0.0 \pm 0.0$ & $0.0 \pm 0.0$ & 12 & 2 & small boulders \\
\hline & 60 & $6.7 \pm 0.6$ & $0.0 \pm 0.0$ & $0.0 \pm 0.0$ & $0.0 \pm 0.0$ & $0.0 \pm 0.0$ & 14.5 & 2 & small boulders \\
\hline 11 & 2 & $11.7 \pm 3.1$ & $0.0 \pm 0.0$ & $0.0 \pm 0.0$ & $0.0 \pm 0.0$ & $0.0 \pm 0.0$ & 7 & 3 & big boulders \\
\hline & 10 & $22.3 \pm 0.6$ & $0.0 \pm 0.0$ & $0.0 \pm 0.0$ & $0.0 \pm 0.0$ & $0.0 \pm 0.0$ & 14.1 & 3 & rock \\
\hline
\end{tabular}


TABLE 1 (Cont.) - Sea urchin density and percent algal cover (mean of three samples \pm standard deviation), water depth, bottom declivity (Bott. decliv.) and substrate type against distance (Dist.) from the tide line for transects 1 to 13 . (Measurements were taken every 10 m until sandy sediments were reached, with the exception of those marked with an asterisk (*). Bottom declivity was measured on a four-level scale as described in the methodology.)

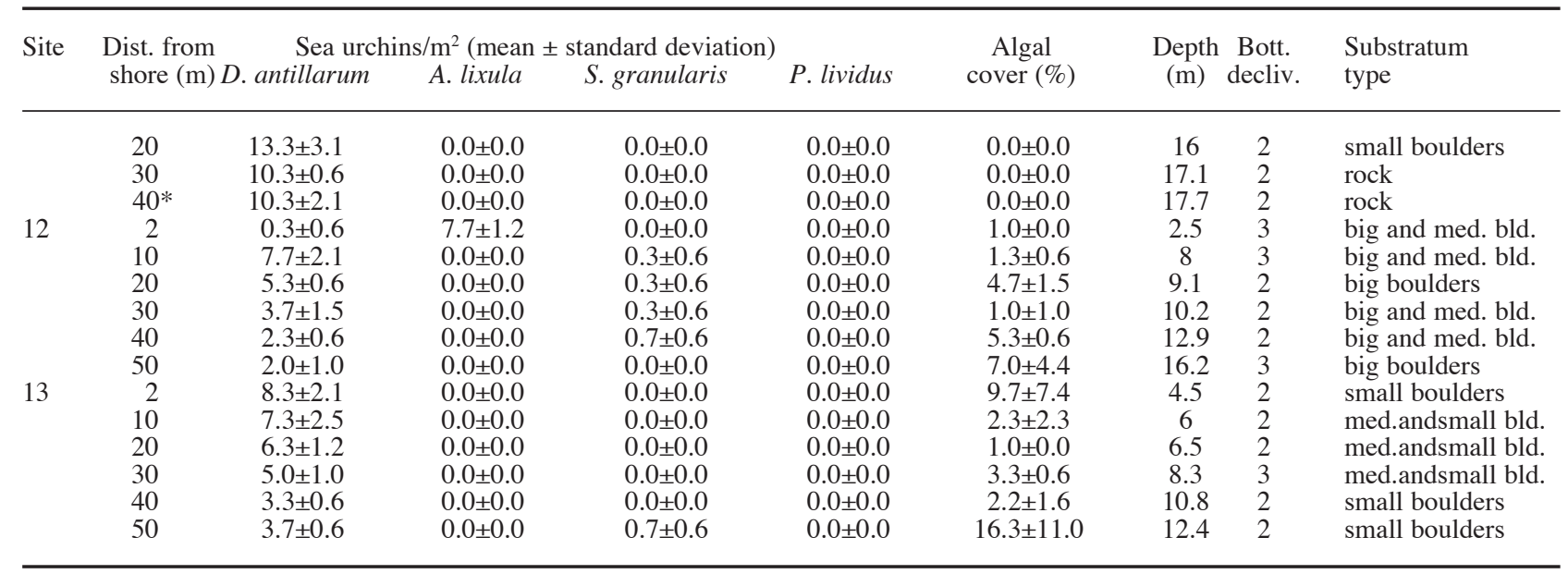

dock, this species was observed at water depths of less than $1 \mathrm{~m}$. In cliff areas (i.e. declivity close to $90^{\circ}$ ) close to the tide line, $D$. antillarum was commonly observed at depths of 1-2 m. On boulder beaches, where declivity is generally low, this species only appeared below 5-7 m water depth.

Arbacia lixula and Paracentrotus lividus preferred shallower depths, being found close to the shore. Sphaerechinus granularis was always found at very low densities and typically occurred 30-40 $\mathrm{m}$ from the shore at depths of approximately $6 \mathrm{~m}$. Two-way ANOVA results show that there were significant differences $(p<0.05)$ between sea urchin densities and water depth, between sea urchin species, and between both these sets of parameters (Table 3). Algal cover did not show a clear trend with distance from shore or water depth (Tables 1 and 2). On average, sandy sediments were found from about $80 \mathrm{~m}$ along transects from the tide line.

\section{Substratum type}

Diadema antillarum, Arbacia lixula and Paracentrotus lividus were found living on almost all recorded substrata, particularly on rock floors and big boulders (Tables 1 and 2). Sphaerechinus granularis occurred mostly on small boulders. In general, seaweed and sea urchins were absent from sandy substrata.

\section{Water turbulence}

Water turbulence was assessed as being level 2 for transects 1-15SE and 22-23SW; level 3 for tran- sect $19 \mathrm{~N}$ (which lies in a sheltered area) and 21SW; and level 4 for transects $16 \mathrm{~N}, 17 \mathrm{~N}, 18 \mathrm{~N}$ and $20 \mathrm{SW}$.

\section{Factors limiting spatial distribution of Diadema antillarum}

All of the environmental factors analysed were integrated in a single non-linear equation. The independent variables selected were: distance from tide line, water depth, and water turbulence. The remaining variables were eliminated since they did not contribute significantly to the adjusted $\mathrm{R}^{2}$. The multiple non-linear regression equation describing the spatial distribution of $D$. antillarum on the rocky shores is:

$$
\begin{aligned}
& \text { D. antillarum } / \mathrm{m}^{2}(\sqrt{ } \sqrt{ })=0.121-0.209 \mathrm{DS}(\sqrt{ } \sqrt{ })+ \\
& 2.052 \mathrm{D}(\sqrt{ } \sqrt{ })-1.778 \mathrm{WT}(\sqrt{ } \sqrt{ })-0.007 \mathrm{WT}^{4}(\sqrt{ } \sqrt{ })
\end{aligned}
$$

where DS is distance from tide line (in $\mathrm{m}$ ), D is water depth (in m), WT is water turbulence categorised according to the four-point scale (defined above) and $\sqrt{ } \sqrt{ }$ indicates data are square-root transformed. The adjusted $\mathrm{R}^{2}$ was $60.99 \%$ and the number of samples (n) used for the equation was 454 . The corresponding ANOVA test $(95 \%$ confidence interval) was significant $(\mathrm{p}<0.05)$.

\section{Sea urchin vs. macroalgae abundance}

In general, sea urchin densities and algal cover were negatively correlated (see Tables 1 and $2 ; \mathrm{R}^{2}$ is $75.6 \%$ ). Due to the predominance of $D$. antillarum among sea urchins, the assessment of sea urchin 
TABLE 2. - Sea urchin density and percent algal cover (mean of three samples \pm standard deviation), water depth, bottom declivity (Bott. decliv.) and substrate type against distance (Dist.) from tide line for transects 14 to 23. (Measurements were made until sandy sediments were found, except those marked with an asterisk (*). Bottom declivity was measured on a four-level scale described in the methodology.)

\begin{tabular}{|c|c|c|c|c|c|c|c|c|c|}
\hline \multirow[t]{2}{*}{ Site } & \multirow{2}{*}{$\begin{array}{c}\text { Dist. from } \\
\text { shore (m) }\end{array}$} & \multicolumn{3}{|c|}{ Sea urchins $/ \mathrm{m}^{2}$ (mean \pm standard deviation $)$} & \multirow[b]{2}{*}{ P.lividus } & \multirow{2}{*}{$\begin{array}{c}\text { Algal } \\
\text { cover }(\%)\end{array}$} & \multirow{2}{*}{$\begin{array}{l}\text { Depth } \\
\text { (m) }\end{array}$} & Bott. & Substratum \\
\hline & & D.antillarum & A.lixula & S.granularis & & & & decliv. & type \\
\hline 14 & 2 & $0.0 \pm 0.0$ & $0.0 \pm 0.0$ & $0.0 \pm 0.0$ & $0.0 \pm 0.0$ & $64.0 \pm 20.5$ & 2.5 & 2 & big and med. bld. \\
\hline & 10 & $14.3 \pm 2.5$ & $0.0 \pm 0.0$ & $0.0 \pm 0.0$ & $0.0 \pm 0.0$ & $0.0 \pm 0.0$ & 3.5 & 2 & big and med. bld. \\
\hline & 20 & $12.0 \pm 3.5$ & $0.0 \pm 0.0$ & $0.0 \pm 0.0$ & $0.0 \pm 0.0$ & $0.0 \pm 0.0$ & 5.4 & 2 & big and med. bld. \\
\hline & 30 & $7.7 \pm 1.5$ & $0.0 \pm 0.0$ & $0.0 \pm 0.0$ & $0.0 \pm 0.0$ & $0.3 \pm 0.6$ & 7.5 & 2 & med.andsmall bld. \\
\hline & 40 & $8.3 \pm 1.5$ & $0.0 \pm 0.0$ & $0.0 \pm 0.0$ & $0.0 \pm 0.0$ & $0.0 \pm 0.0$ & 7.9 & 2 & med.andsmall bld. \\
\hline & 50 & $8.0 \pm 1.0$ & $0.0 \pm 0.0$ & $0.0 \pm 0.0$ & $0.0 \pm 0.0$ & $2.7 \pm 2.1$ & 8.4 & 2 & med.andsmall bld. \\
\hline & 60 & $6.7 \pm 0.6$ & $0.0 \pm 0.0$ & $0.0 \pm 0.0$ & $0.0 \pm 0.0$ & $0.0 \pm 0.0$ & 11.3 & 3 & med.andsmall bld. \\
\hline & 70 & $4.7 \pm 1.5$ & $0.0 \pm 0.0$ & $0.0 \pm 0.0$ & $0.0 \pm 0.0$ & $2.0 \pm 1.0$ & 17.6 & 3 & med.andsmall bld. \\
\hline 15 & 2 & $0.7 \pm 1.2$ & $0.0 \pm 0.0$ & $0.0 \pm 0.0$ & $0.0 \pm 0.0$ & $88.3 \pm 7.6$ & 5.9 & 2 & rock \\
\hline & 10 & $0.3 \pm 0.6$ & $0.0 \pm 0.0$ & $0.0 \pm 0.0$ & $0.0 \pm 0.0$ & $67.0 \pm 57.2$ & 7.8 & 2 & big and med. bld. \\
\hline & 20 & $1.3 \pm 1.5$ & $0.0 \pm 0.0$ & $0.0 \pm 0.0$ & $0.0 \pm 0.0$ & $34.0 \pm 57.2$ & 8.2 & 3 & big boulders \\
\hline & 30 & $2.0 \pm 2.0$ & $0.0 \pm 0.0$ & $0.0 \pm 0.0$ & $0.0 \pm 0.0$ & $26.3 \pm 43.9$ & 10.3 & 2 & big boulders \\
\hline 16 & 2 & $0.0 \pm 0.0$ & $0.0 \pm 0.0$ & $0.0 \pm 0.0$ & $0.0 \pm 0.0$ & $73.7 \pm 5.5$ & 4.1 & 1 & med.andsmall bld. \\
\hline & 10 & $0.0 \pm 0.0$ & $0.3 \pm 0.6$ & $0.0 \pm 0.0$ & $0.0 \pm 0.0$ & $43.0 \pm 10.8$ & 4.4 & 1 & med.andsmall bld. \\
\hline & 20 & $0.0 \pm 0.0$ & $1.0 \pm 1.7$ & $0.0 \pm 0.0$ & $0.0 \pm 0.0$ & $78.7 \pm 5.9$ & 4.9 & 1 & med.andsmall bld. \\
\hline & 30 & $0.0 \pm 0.0$ & $0.0 \pm 0.0$ & $0.0 \pm 0.0$ & $0.0 \pm 0.0$ & $64.7 \pm 7.8$ & 6.2 & 1 & med.andsmall bld. \\
\hline & 40 & $0.0 \pm 0.0$ & $0.7 \pm 1.2$ & $0.0 \pm 0.0$ & $0.0 \pm 0.0$ & $39.3 \pm 1.5$ & 6.8 & 1 & med.andsmall bld. \\
\hline & 50 & $0.0 \pm 0.0$ & $0.0 \pm 0.0$ & $0.0 \pm 0.0$ & $0.0 \pm 0.0$ & $40.7 \pm 1.5$ & 6.9 & 1 & med.andsmall bld. \\
\hline 17 & 10 & $0.0 \pm 0.0$ & $0.0 \pm 0.0$ & $0.0 \pm 0.0$ & $0.0 \pm 0.0$ & $7.7 \pm 6.7$ & 3.4 & 1 & med.andsmall bld. \\
\hline & 20 & $0.0 \pm 0.0$ & $0.0 \pm 0.0$ & $0.0 \pm 0.0$ & $0.0 \pm 0.0$ & $24.3 \pm 21.5$ & 3.6 & 1 & med.andsmall bld. \\
\hline & 30 & $0.0 \pm 0.0$ & $0.3 \pm 0.6$ & $0.0 \pm 0.0$ & $0.0 \pm 0.0$ & $25.7 \pm 22.5$ & 4.1 & 2 & med.andsmall bld. \\
\hline & 40 & $0.0 \pm 0.0$ & $0.0 \pm 0.0$ & $0.0 \pm 0.0$ & $0.0 \pm 0.0$ & $40.3 \pm 30.2$ & 6.3 & 1 & big and med. bld. \\
\hline & 50 & $0.0 \pm 0.0$ & $0.0 \pm 0.0$ & $0.0 \pm 0.0$ & $0.0 \pm 0.0$ & $49.3 \pm 32.5$ & 6.5 & 1 & big and med. bld. \\
\hline & 60 & $0.0 \pm 0.0$ & $0.0 \pm 0.0$ & $0.0 \pm 0.0$ & $0.0 \pm 0.0$ & $37.0 \pm 28.8$ & 6.6 & 1 & big and med. bld. \\
\hline & 70 & $0.0 \pm 0.0$ & $1.0 \pm 1.7$ & $0.0 \pm 0.0$ & $0.0 \pm 0.0$ & $32.7 \pm 9.7$ & 7.5 & 2 & big and med. bld. \\
\hline & 80 & $0.0 \pm 0.0$ & $1.0 \pm 1.0$ & $0.0 \pm 0.0$ & $0.0 \pm 0.0$ & $40.7 \pm 33.6$ & 8.2 & 2 & big and med. bld. \\
\hline & 90 & $0.0 \pm 0.0$ & $0.3 \pm 0.6$ & $0.0 \pm 0.0$ & $0.0 \pm 0.0$ & $97.7 \pm 1.2$ & 9 & 2 & big and med. bld. \\
\hline & $100 *$ & $0.0 \pm 0.0$ & $0.0 \pm 0.0$ & $0.0 \pm 0.0$ & $0.0 \pm 0.0$ & $96.3 \pm 1.5$ & 10.1 & 2 & big and med. bld. \\
\hline 18 & 2 & $0.0 \pm 0.0$ & $0.0 \pm 0.0$ & $0.0 \pm 0.0$ & $0.0 \pm 0.0$ & $68.0 \pm 2.6$ & 5.5 & 1 & big and med. bld. \\
\hline & 10 & $0.0 \pm 0.0$ & $0.0 \pm 0.0$ & $0.0 \pm 0.0$ & $0.0 \pm 0.0$ & $97.0 \pm 2.0$ & 5.3 & 1 & big and med. bld. \\
\hline & 20 & $0.0 \pm 0.0$ & $0.0 \pm 0.0$ & $0.0 \pm 0.0$ & $0.0 \pm 0.0$ & $97.7 \pm 1.5$ & 5.2 & 1 & big and med. bld. \\
\hline & 30 & $0.0 \pm 0.0$ & $0.0 \pm 0.0$ & $0.0 \pm 0.0$ & $0.0 \pm 0.0$ & $67.0 \pm 2.6$ & 5.4 & 1 & big and med. bld. \\
\hline & 40 & $0.0 \pm 0.0$ & $0.0 \pm 0.0$ & $0.0 \pm 0.0$ & $0.0 \pm 0.0$ & $84.7 \pm 6.5$ & 5.8 & 1 & big and med. bld. \\
\hline & 50 & $0.0 \pm 0.0$ & $0.0 \pm 0.0$ & $0.0 \pm 0.0$ & $0.0 \pm 0.0$ & $37.0 \pm 4.0$ & 7.2 & 2 & med.andsmall bld. \\
\hline & 60 & $0.0 \pm 0.0$ & $0.0 \pm 0.0$ & $0.0 \pm 0.0$ & $0.0 \pm 0.0$ & $35.7 \pm 4.7$ & 7.3 & 2 & med.andsmall bld. \\
\hline & 70 & $0.0 \pm 0.0$ & $0.0 \pm 0.0$ & $0.0 \pm 0.0$ & $0.0 \pm 0.0$ & $95.0 \pm 3.0$ & 8 & 2 & big and med. bld. \\
\hline & 80 & $0.0 \pm 0.0$ & $0.0 \pm 0.0$ & $0.0 \pm 0.0$ & $0.0 \pm 0.0$ & $92.3 \pm 1.5$ & 8.5 & 2 & big and med. bld. \\
\hline & 90 & $0.3 \pm 0.6$ & $0.0 \pm 0.0$ & $0.0 \pm 0.0$ & $0.0 \pm 0.0$ & $97.3 \pm 2.1$ & 10.3 & 2 & big boulders \\
\hline & 100 & $4.7 \pm 2.1$ & $0.0 \pm 0.0$ & $0.0 \pm 0.0$ & $0.0 \pm 0.0$ & $2.0 \pm 1.0$ & 10.2 & 2 & big boulders \\
\hline & 110 & $0.0 \pm 0.0$ & $0.0 \pm 0.0$ & $0.0 \pm 0.0$ & $0.0 \pm 0.0$ & $95.7 \pm 2.1$ & 11.7 & 2 & big boulders \\
\hline 19 & 2 & $6.7 \pm 3.2$ & $0.0 \pm 0.0$ & $1.3 \pm 0.6$ & $0.0 \pm 0.0$ & $1.0 \pm 0.0$ & 7.2 & 2 & med.andsmall bld. \\
\hline & 10 & $4.7 \pm 0.6$ & $0.0 \pm 0.0$ & $0.3 \pm 0.6$ & $0.0 \pm 0.0$ & $1.0 \pm 0.0$ & 8.5 & 2 & med.andsmall bld. \\
\hline & 20 & $3.0 \pm 1.0$ & $0.0 \pm 0.0$ & $0.3 \pm 0.6$ & $0.0 \pm 0.0$ & $1.0 \pm 0.0$ & 9.9 & 2 & small boulders \\
\hline & 30 & $2.0 \pm 1.0$ & $0.0 \pm 0.0$ & $1.3 \pm 0.6$ & $0.0 \pm 0.0$ & $1.0 \pm 0.0$ & 11.1 & 2 & small boulders \\
\hline & 40 & $2.3 \pm 1.5$ & $0.0 \pm 0.0$ & $0.7 \pm 0.6$ & $0.0 \pm 0.0$ & $0.0 \pm 0.0$ & 13.5 & 2 & small boulders \\
\hline 20 & 10 & $0.0 \pm 0.0$ & $0.0 \pm 0.0$ & $0.0 \pm 0.0$ & $0.0 \pm 0.0$ & $97.7 \pm 2.5$ & 1.8 & 2 & big and med. bld. \\
\hline & 20 & $0.0 \pm 0.0$ & $0.0 \pm 0.0$ & $0.0 \pm 0.0$ & $0.0 \pm 0.0$ & $93.3 \pm 11.5$ & 3.1 & 2 & big and med. bld. \\
\hline & 30 & $0.0 \pm 0.0$ & $0.0 \pm 0.0$ & $0.0 \pm 0.0$ & $0.0 \pm 0.0$ & $100.0 \pm 0.0$ & 5.2 & 1 & big and med. bld. \\
\hline & 40 & $0.0 \pm 0.0$ & $0.0 \pm 0.0$ & $0.0 \pm 0.0$ & $0.0 \pm 0.0$ & $100.0 \pm 0.0$ & 5.3 & 1 & big and med. bld. \\
\hline & 50 & $0.0 \pm 0.0$ & $0.0 \pm 0.0$ & $0.0 \pm 0.0$ & $0.0 \pm 0.0$ & $100.0 \pm 0.0$ & 4.8 & 1 & big and med. bld. \\
\hline & 60 & $0.0 \pm 0.0$ & $0.0 \pm 0.0$ & $0.0 \pm 0.0$ & $0.0 \pm 0.0$ & $100.0 \pm 0.0$ & 4.7 & 1 & big and med. bld. \\
\hline & 70 & $0.0 \pm 0.0$ & $0.0 \pm 0.0$ & $0.0 \pm 0.0$ & $0.0 \pm 0.0$ & $100.0 \pm 0.0$ & 7.3 & 2 & big and med. bld. \\
\hline & 80 & $0.0 \pm 0.0$ & $0.0 \pm 0.0$ & $0.0 \pm 0.0$ & $0.0 \pm 0.0$ & $100.0 \pm 0.0$ & 8.5 & 2 & big and med. bld. \\
\hline & 90 & $0.0 \pm 0.0$ & $0.0 \pm 0.0$ & $0.0 \pm 0.0$ & $0.0 \pm 0.0$ & $100.0 \pm 0.0$ & 9.7 & 2 & big and med. bld. \\
\hline & 100 & $0.0 \pm 0.0$ & $0.0 \pm 0.0$ & $0.0 \pm 0.0$ & $0.0 \pm 0.0$ & $100.0 \pm 0.0$ & 10 & 2 & big and med. bld. \\
\hline & 110 & $0.0 \pm 0.0$ & $0.0 \pm 0.0$ & $0.0 \pm 0.0$ & $0.0 \pm 0.0$ & $100.0 \pm 0.0$ & 10.2 & 2 & big and med. bld. \\
\hline & 120 & $0.0 \pm 0.0$ & $0.0 \pm 0.0$ & $0.0 \pm 0.0$ & $0.0 \pm 0.0$ & $100.0 \pm 0.0$ & 10.5 & 2 & big and med. bld. \\
\hline & 130 & $0.0 \pm 0.0$ & $0.0 \pm 0.0$ & $0.0 \pm 0.0$ & $0.0 \pm 0.0$ & $100.0 \pm 0.0$ & 11.4 & 2 & big and med. bld. \\
\hline & 140 & $0.7 \pm 1.2$ & $0.0 \pm 0.0$ & $0.0 \pm 0.0$ & $0.0 \pm 0.0$ & $67.3 \pm 56.6$ & 12.8 & 2 & big and med. bld. \\
\hline & $150 *$ & $0.0 \pm 0.0$ & $0.0 \pm 0.0$ & $0.0 \pm 0.0$ & $0.0 \pm 0.0$ & $100.0 \pm 0.0$ & 13.1 & 2 & big and med. bld. \\
\hline 21 & 2 & $0.0 \pm 0.0$ & $0.0 \pm 0.0$ & $0.0 \pm 0.0$ & $0.0 \pm 0.0$ & $86.7 \pm 3.8$ & 2.8 & 2 & small boulders \\
\hline & 10 & $0.0 \pm 0.0$ & $0.0 \pm 0.0$ & $0.0 \pm 0.0$ & $0.0 \pm 0.0$ & $83.0 \pm 3.0$ & 4.1 & 2 & small boulders \\
\hline 22 & 2 & $0.0 \pm 0.0$ & $0.0 \pm 0.0$ & $0.0 \pm 0.0$ & $0.0 \pm 0.0$ & $75.7 \pm 30.1$ & 3.4 & 1 & big and med. bld. \\
\hline & 10 & $0.0 \pm 0.0$ & $13.7 \pm 16.5$ & $0.0 \pm 0.0$ & $0.0 \pm 0.0$ & $63.0 \pm 52.8$ & 3.7 & 1 & big and med. bld. \\
\hline & 20 & $0.0 \pm 0.0$ & $3.3 \pm 5.8$ & $0.0 \pm 0.0$ & $0.0 \pm 0.0$ & $64.3 \pm 54.8$ & 4.3 & 2 & big and med. bld. \\
\hline & 30 & $0.3 \pm 0.6$ & $1.3 \pm 2.3$ & $0.0 \pm 0.0$ & $0.0 \pm 0.0$ & $88.3 \pm 15.9$ & 8 & 3 & big and med. bld. \\
\hline & 40 & $0.7 \pm 1.2$ & $0.0 \pm 0.0$ & $0.7 \pm 1.2$ & $0.0 \pm 0.0$ & $53.7 \pm 45.6$ & 8.5 & 2 & big and med. bld. \\
\hline 23 & 2 & $0.0 \pm 0.0$ & $0.0 \pm 0.0$ & $0.0 \pm 0.0$ & $0.0 \pm 0.0$ & $0.0 \pm 0.0$ & 1 & 2 & big and med. bld. \\
\hline & 10 & $0.0 \pm 0.0$ & $0.7 \pm 1.2$ & $0.0 \pm 0.0$ & $0.0 \pm 0.0$ & $20.0 \pm 34.6$ & 1.8 & 2 & big and med. bld. \\
\hline & 20 & $0.0 \pm 0.0$ & $0.0 \pm 0.0$ & $0.0 \pm 0.0$ & $0.0 \pm 0.0$ & $74.7 \pm 37.8$ & 3.1 & 2 & big and med. bld. \\
\hline & 30 & $0.0 \pm 0.0$ & $0.0 \pm 0.0$ & $0.0 \pm 0.0$ & $0.0 \pm 0.0$ & $86.7 \pm 7.1$ & 5.3 & 2 & big and med. bld. \\
\hline & 40 & $0.0 \pm 0.0$ & $0.0 \pm 0.0$ & $0.0 \pm 0.0$ & $0.0 \pm 0.0$ & $51.3 \pm 32.3$ & 5.5 & 2 & big and med. bld. \\
\hline & 50 & $0.7 \pm 0.6$ & $0.0 \pm 0.0$ & $0.0 \pm 0.0$ & $0.0 \pm 0.0$ & $2.0 \pm 2.0$ & 6.9 & 2 & big and med. bld. \\
\hline & 60 & $1.3 \pm 0.6$ & $0.0 \pm 0.0$ & $0.0 \pm 0.0$ & $0.0 \pm 0.0$ & $0.0 \pm 0.0$ & 10.8 & 3 & big and med. bld. \\
\hline & 70 & $0.7 \pm 0.6$ & $0.0 \pm 0.0$ & $0.0 \pm 0.0$ & $0.0 \pm 0.0$ & $0.0 \pm 0.0$ & 12 & 3 & big and med. bld. \\
\hline
\end{tabular}


TABLE 3. - Results of two-way ANOVA test of water depth and the densities of four sea urchin species. $(\mathrm{df}=$ Degrees of freedom, MS $=$ Mean Square.)

\begin{tabular}{lrrrrrr}
\hline \multicolumn{3}{r}{$\begin{array}{r}\text { Statistic Two-Way ANOVA } \\
\text { Effect df Effect }\end{array}$} & \multicolumn{5}{c}{ 1-Depth, 2-Sea urchins } \\
& & & & \\
& & & & & & \\
1 & 20 & 16.78 & 1812 & 10.35 & 1.62 & 0.0401 \\
2 & 3 & 994.14 & 1812 & 10.35 & 96.08 & 0.0000 \\
12 & 60 & 28.51 & 1812 & 10.35 & 2.76 & $3.9 \mathrm{E}-11$ \\
\hline
\end{tabular}

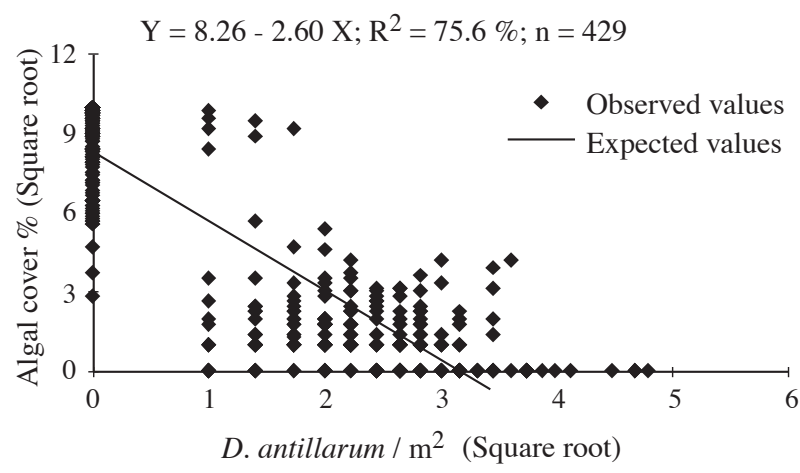

FIG. 2. - Linear regression between the density of Diadema antillarum and algal cover. Percent algal cover (square-root transformed $)=8.26-2.60$ Diadema antillarum $/ \mathrm{m}^{2}$ (square-root trans formed); $\mathrm{R}^{2}=75.6 \% ; \mathrm{n}=429$.

density against macroalgae abundance focuses upon this species.

High algal cover was found only in those areas where $D$. antillarum was absent. However, with densities of 1 individual $/ \mathrm{m}^{2}$ or higher, the percentage of algal cover decreased markedly and the linear regression between the percentage of algal cover and $D$. antillarum density shows an inverse relationship (Fig. 2). The linear regression equation is:

$$
\mathrm{A}(\sqrt{ } \sqrt{ })=8.26-2.60 \mathrm{D}(\sqrt{ } \sqrt{ })
$$

where $\mathrm{A}$ is the percentage of algal cover, $\mathrm{D}$ is the number of individuals of Diadema antillarum per square metre, and $\sqrt{ } \sqrt{ }$ indicates data are square-root transformed. The adjusted $\mathrm{R}^{2}$ is $75.6 \%$, and the number of observations is 429 .

\section{DISCUSSION}

Among the less abundant sea urchin species, Sphaerechinus granularis reached its higher densities at $30-40 \mathrm{~m}$ from shore at depths of approximately $6 \mathrm{~m}$. Similar results were found by Maciel and Gonçalves (1998) at the Azores Islands, and this is probably related to the lower water turbulence that occurs at this depth. Diadema antillarum exhibits aggressive behaviour towards other urchin species (Shulman, 1990), which might explain the low densities of $S$. granularis in the vicinity of high densities of D. antillarum.

The other less abundant sea urchin species found in this study were P. lividus and A. lixula, showing a very similar distribution in terms of water depth. One possible explanation for their distribution in shallower water is the distribution of their prey species: Cystoseira spp., which live in very shallow waters off Madeira (Levring, 1974; Augier, 1985; Morton et al., 1998), and appear to be the preferred diet of these sea urchin species (Bergin, 1987; Benedetti-Cecchi and Cinelli, 1995). However, where D. antillarum is absent (i.e. along the major northern coastal area) A. lixula were found down to $9 \mathrm{~m}$, suggesting that their restricted distribution might also be mediated by competition with $D$. antillarum. Shulman (1990) recorded similar observations with the $D$. antillarum and Keller (1983) with other sea urchin species in the Caribbean reefs.

High densities of $D$. antillarum (up to a maximum record of 68 individuals $/ \mathrm{m}^{2}$ ) were observed during this study, and similar densities have been recorded in Jamaica by other authors (e.g. Sammarco, 1980; Sammarco and Williams, 1982). It is generally accepted that human activities are responsible for high sea urchin densities because of the removal of predators through over-fishing (e.g. Mann, 1977; Hughes, 1994; Casañas et al., 1998; Sala et al., 1998; Steneck, 1998). Nevertheless, on the basis of paleontological and early historical reports, Jackson (1997) considered D. antillarum to have always been the most abundant sea urchin in the Caribbean. At Madeira Island, adult D. antillarum do not have any specific predator, which may be related to over-fishing. According to Carpenter (1984), high sea urchin densities, together with the absence of predators, may explain the frequent presence of $D$. antillarum on exposed rocks, a phenomenon observed on Madeira's rocky shores, which contrasts with the "homing" behaviour described by Carpenter (1990).

In this study, algal cover was not considered a limiting factor for $D$. antillarum distribution, but rather a result of the urchin's presence. We found evidence of two distinct and alternative rocky shore communities off Madeira Island. Where D. antillarum occurred in large numbers, algae were prevented from reaching any great abundance, where- 
as sea urchins were not present where algae formed a dense cover. We suggest that a dense cover of algae does not prevent $D$. antillarum from occurring in high densities, but that there are other limiting factors (e.g. water turbulence) that do so. However, Wirtz (1995) considers that the types of community structure observed at Madeira Island are an example of alternative stable states, with shifts occurring only after catastrophic events. The negative relationship observed between the percentage of algal cover and the density of $D$. antillarum has been previously described in Caribbean waters by Carpenter (1981), Sammarco (1982a) and Johnson and Mann (1993).

The predominantly rough waters off the north and west coasts of Madeira Island (Instituto Hidrográfico, 1979; Abreu and Biscoito, 1998; Caldeira and Lekou, 2000) may be responsible for differences in D. antillarum abundance between the north and south coasts. The species was absent from many shallow areas, where wave action has its greatest impact, but in places where there is no wave action, such as in the Porto Santo dock, D. antillarum were found to occur at depths of less than $1 \mathrm{~m}$. Weil et al. (1984) and Casañas et al. (1998) have also recorded this species in shallow waters on a Venezuelan coral reef and at the Canary Islands respectively.

For substratum type, the results showed that $D$. antillarum was found living in high densities on several kinds of rocky shores, mainly preferring rock platforms and, to a lesser extent, areas of large boulders. However, sandy sediments are avoided and limit their habitat.

Bottom declivity is linked to several of the other factors considered. It affects water depth and consequently exposure to water turbulence, which occurs mainly in shallow waters. For example, in cliff areas, where declivity is around $90^{\circ}, D$. antillarum appeared at depths of 1-2 m, whereas on boulder beaches, where declivity is low, it appeared only below 5-7 m, and this distribution pattern may be dependent upon the degree of water turbulence.

The multiple non-linear regression resulting from our study reveals that distance from the tide line, together with water depth and water turbulence, explained $60.99 \%$ of the observed sea urchin distribution. We propose four possible explanations for this low adjusted $R^{2}$ value. (1) Sea urchin densities were measured from July to August, while the water turbulence scale is an annual average, which may account for some of the variation in this depen- dent variable. (2) Although previous studies have suggested some factors that might explain the distribution of $D$. antillarum, there are other factors that might have equal importance, such as the aggressive behaviour of territorial pomacentrid fishes (Sammarco and Williams, 1982), and the density of other invertebrate species (Casañas et al., 1998). (3) Neither recruits nor juveniles (test diameter $<10 \mathrm{~mm}$ ) were found during the sampling months, and the inclusion of both recruits and juveniles in addition to adults should be considered in future studies since this may increase the adjusted $\mathrm{R}^{2}$ value. (4) D. antillarum is highly adaptable to different environmental conditions and inhabits a number of different marine communities: shallow coral reefs, rocky shores, seagrass beds, sandy bottoms, mangrove roots, and even water depths down to $400 \mathrm{~m}$ (Weil et al., 1984). Diadema antillarum has a variety of successful adaptive strategies (Ebert, 1985), including the ability to increase the relative size of the Aristotle's lantern compared to the size of the test when food is limited. Moreover, high population density leads to reduced body size and gamete production; body size can be adjusted quickly (by positive or negative growth) to a given resource or density-dependent factors (Levitan, 1988, 1989, 1991a, b). Populations using this life history strategy tend to closely track environmental carrying capacity (Levitan, 1989).

Another possible adaptive theory to explain the high $D$. antillarum densities on the "blanquizal" (bare-ground) areas observed off the southeast coast of Madeira involves its feeding behaviour. Though it is preferentially herbivorous (Sammarco, 1982a, b; Kullen, 1996), D. antillarum can also be omnivorous (Carpenter, 1981), in what Lawrence (1975) considers as opportunistic feeding behaviour. Absorption of dissolved nutrients by the spines has been observed in P. lividus (De Burgh, 1975; Anonymous, 1996) and is yet another possible adaptive theory.

Future investigations into the spatial distribution of $D$. antillarum should consider additional variables, such as temperature, aggressive territorial pomacentrid fishes, density of all invertebrate species, surface of the substratum, the number and size of refuges, local current velocity, and dissolved nutrients in the water. Further studies on the ecology of $D$. antillarum would be of great interest and would contribute towards a better understanding and management of the rocky shore ecosystems of Madeira Island. 


\section{ACKNOWLEDGEMENTS}

Special thanks are due to the staff of the Museu Municipal do Funchal, Universidade da Madeira, Parque Natural da Madeira and Fundação Berardo. Thanks are also due to Doutor Manfred Kaufman, Doutor Thomas Dellinger, Doutor Peter Wirtz, Luís Costa, Cláudia, Roberto, Boaventura, Filipe, Dra Mafalda, Dra Rosa Pires, Dra Carla Renato, Dra Délia Cravo and Artur Silva.

\section{REFERENCES}

Abreu, A.D., R. Araújo, M.J. Biscoito, M.A. Malaquias and P.N. Gomes. - 1995. Fauna Marinha da Madeira. Jornal da Madeira Magazine, Funchal.

Abreu, A.D. and M.J. Biscoito. - 1998. A vida nos mares da Madeira. Islenha, 23: 5-14.

Andrew, N.L. - 1989. Contrasting ecological implications of food limitation in sea urchins and herbivorous gastropods. Mar. Ecol. Prog. Ser., 51: 189-193.

Anonymous. - 1996. Os ouriços do mar. Notícias do Mar, 136: 1-25.

Augier, H. - 1985. Première contribution à l'étude et à la cartographie des biocenoses marines benthiques de l'Ile de Madère. Bol. Mus. Mun. Funchal, 37(168): 86-129.

Baez, M. - 1993. Origins and affinities of the fauna of Madeira. Bol. Mus. Mun. Funchal, Suppl. 2: 9-40.

Bauer, J.C. - 1980. Observations on geographical variations in population density of the echinoid Diadema antillarum within the western North Atlantic. Bull. Mar. Sci., 30(2): 509-515.

Benedetti-Cecchi, L. and F. Cinelli. - 1995. Habitat heterogeneity, sea urchin grazing and the distribution of algae in littoral rock pools on the west coast of Italy (Western Mediterranean). Mar. Ecol. Prog. Ser., 126: 203-212.

Bergin, F. - 1987. Contenus digestifs de Paracentrotus lividus et d'Arbacia lixula dans la region d'el Dabba (Egypte). In: C.F. Boudouresque (ed.), Colloque international sur Paracentrotus lividus et les oursins comestibles, pp. 107-116. GIS Posidonie publ., Marseille.

Beyhl, F.E., B. Mies and P. Ohm. - 1995. Macaronesia - a biogeographical puzzle. Bol. Mus. Mun. Funchal, Suppl. 4: 107-113.

Bianchi, C.N., C. Morri, G. Sartoni and P. Wirtz. - 1998. Sublittoral epibenthic communities around Funchal (Ilha da Madeira, NE Atlantic). Bol. Mus. Mun. Funchal, Suppl. 5: 59-80.

Biscoito, M.J. and A.D. Abreu. - 1998. Os mares. Região Autónoma da Madeira. Expo 98 ed., Lisboa.

Caldeira, R.M.A. and S. Lekou. - 2000. Madeira, um oásis no Atlântico - Uma introdução aos estudos oceanográficos no arquipélago da Madeira. D.R. Regional de Formação Profissional, Madeira.

Carpenter, R.C. - 1981. Grazing by Diadema antillarum (Philippi) and its effects on the benthic algal community. J. Mar. Res., 39: 749-765.

Carpenter, R.C. - 1984. Predator and population density control of homing behaviour in the Caribbean echinoid Diadema antillarum. Mar. Biol., 82: 101-108.

Carpenter, R.C. - 1990. Mass mortality of Diadema antillarum I. Long-term effects on sea urchins population-dynamics and coral reef algal communities. Mar. Biol., 104: 67-77.

Casañas, A., H.H. Larsen and R. Haroun. - 1998. Ecological succession in subtidal waters of Canary Islands dominated by the sea urchin Diadema antillarum. Bol. Mus. Mun. Funchal, Suppl. 5: 139-146.

De Burgh. - 1975. Aspects of the absorption of dissolved nutrients by spines of Paracentrotus lividus (Lamarck). Ph. D. thesis, Univ. Dublin, Ireland.

Ebert, T.A. - 1985. Sensitivity of fitness to macroparameter changes: an analysis of survivorship and individual growth in sea urchin life histories. Oecologia, 65: 461-467.

Falcón, J.M., S.A. Bortone, A. Brito and C.M. Bundrick. - 1996.
Structure of and relationships within and between the littoral, rock-substrate fish communities off four islands in the Canarian Archipelago. Mar. Biol., 125: 215-231.

Fernandez, C. and C.F. Boudouresque. - 1997. Phenotypic plasticity of Paracentrotus lividus (Echinodermata: Echinoidea) in a lagoonal environment. Mar. Ecol. Prog. Ser., 152: 145-154.

Hughes, T.P. - 1994. Catastrophes, phase shifts, and large-scale degradation of a Caribbean coral reef. Science, 265: 1547-1551.

Instituto Hidrográfico. - 1979. Roteiro do arquipélago da Madeira e Ilhas Selvagens. 2. ${ }^{a}$ Edição, Instituto Hidrográfico, Lisboa.

Jackson, J.B.C. - 1997. Reefs since Columbus. Coral Reefs, 16: S23-S32.

Johnson, C.R. and K.H. Mann. - 1993. Rapid succession in subtidal understorey seaweeds during recovery from overgrazing by sea urchins in Eastern Canada. Bot. Mar., 36: 63-77.

Keller, B.D. - 1983. Co-existence of sea urchins in seagrass meadows: an experimental analysis of competition and predation. Ecology, 64: 1581-1598.

Kempf, M. - 1962. Recherches d'écologie comparée sur Paracentrotus lividus (Lmk.) et Arbacia lixula (L.). Rec. Trav. Stat. Mar. Endoume, 25(39): 47-116.

Kullen, M. - 1996. The effects of the 1983 mass mortality of the sea urchin Diadema antillarum on coral reef ecosystems of the Caribbean. Thesis, Georgetown Univ., Washington D.C.

Lawrence, J.M. - 1975. On the relationships between marine plants and sea urchins. Oceanogr. Mar. Biol. Ann. Ver., 13: 213-286.

Lessios, H.A. - 1988a. Mass mortality of Diadema antillarum in the Caribbean: what have we learned? Ann. Rev. Ecol. Syst., 19: 371-393.

Lessios, H.A. - 1988b. Population dynamics of Diadema antillarum (Echinodermata: Echinoidea) following mass mortality in Panama. Mar. Biol., 99: 515-526.

Lessios, H.A. - 1995. Diadema antillarum 10 years after mass mortality: still rare, despite help from a competitor. Proc. R. Soc. Lond. B, 259: 331-337.

Levitan, D.R. - 1988. Density-dependent size regulation and negative growth in the sea urchin Diadema antillarum Philippi. Oecologia, 76: 627-629.

Levitan, D.R. - 1989. Density-dependent size regulation in Diadema antillarum: effects on fecundity and survivorship. Ecology, 70: $1414-1424$

Levitan, D.R. - 1991a. Skeletal changes in the test and jaws of the sea urchin Diadema antillarum in response to food limitation. Mar. Biol., 111: 431-435.

Levitan, D.R. - 1991b. Influence of body size and population density on fertilization success and reproductive output in a freespawning invertebrate. Biol. Bull., 181: 261-268.

Levring, T. - 1974. The marine algae of the archipelago of Madeira. Bol. Mus. Mun. Funchal, 28(125): 5-111.

Liddell, W.D. and S.L. Ohlhorst. - 1986. Changes in benthic community composition following the mass mortality of Diadema at Jamaica. J. Exp. Mar. Biol. Ecol., 95: 271-278.

Liddell, W.D. and S.L. Ohlhorst. - 1992. Ten years of disturbance and change on a Jamaican fringing reef. Proceedings of the Seventh International Coral Reef Symposium, Guam, 144-150.

Maciel, P.C. and J.M. Gonçalves. - 1998. Distribuição dos ouriçosdo-mar de espinhos curtos Sphaerechinus granularis na costa da Ilha do Faial, Açores, no Verão de 1996. Third Symposium of Fauna and Flora of the Atlantic Islands, Ponta Delgada, Azores. Abstract book, 182 pp.

Mann, K.H. - 1977. Destruction of kelp-beds by sea urchins: a cyclical phenomenon or irreversible degradation. Helgoländer wiss. Meeresunters, 30: 455-467.

Morton, B., J.C. Briton and A.M.F. Martins. - 1998. Ecologia costeira dos Açores. Sociedade Afonso Chaves, Ponta Delgada, Portugal.

Sala, E., C.F. Boudouresque and M. Harmelin-Vivien. - 1998. Fishing, trophic cascades, and the structure of algal assemblages: evaluation of an old but untested paradigm. Oikos, 82: 425-439.

Sammarco, P.W. - 1980. Diadema and its relationship to coral spat mortality: grazing, competition and biological disturbance. $J$. Exp. Mar. Biol. Ecol., 45: 245-272.

Sammarco, P.W. - 1982a. Effects of grazing by Diadema antillarum Philippi (Echinodermata: Echinoidea) on algal diversity and community structure. J. Exp. Mar. Biol. Ecol., 65: 83-105.

Sammarco, P.W. - 1982b. Echinoid grazing as a structuring force in coral communities: whole reef manipulations. J. Exp. Mar. Biol. Ecol., 61: 31-55. 
Sammarco, P.W., J.S. Levinton and J.O. Ogden. - 1974. Grazing and control of community structure by Diadema antillarum Philippi (Echinodermata Echinoidea): a preliminary study. $J$. Mar. Res., 32: 47-53.

Sammarco, P.W. and A.H. Williams. - 1982. Damselfish territoriality: influence on Diadema distribution and implications for coral community structure. Mar. Ecol. Prog. Ser., 8: 53-59.

Shulman, M.J. - 1990. Aggression among sea urchins on Caribbean coral reefs. J. Exp. Mar. Biol. Ecol., 140: 197-207.

Steneck, R.S. - 1998. Human influences on coastal ecosystems: does overfishing create trophic cascades? Trends Ecol. Evol., 13: 429-430.

Stock, J.H. - 1995. Biogeography and evolutionary scenario of aquatic organisms in Macaronesia. Bol. Mus. Mun. Funchal, Suppl. 4: 729-745.

Turon, X., G. Giribet, S. López and C. Palacín. - 1995. Growth and population structure of Paracentrotus lividus (Echinodermata: Echinoidea) in two contrasting habitats. Mar. Ecol. Prog. Ser., 122: 193-204

Vadas, R.L. - 1990. Comparative foraging behaviour of tropical and boreal sea urchins. In: R.N. Hughes (ed.), Behavioural Mechanisms of Food Selection, pp. 477-514. Springer-Verlag, Berlin.

Vadas, R.L. and R.W. Elner. - 1992. Plant-animal interactions in the north-west Atlantic. In: D.M. John, S.J. Hawkins and J.H. Price (eds.) Plant-animal interactions in the Marine Benthos, pp. 33-60. Clarendon Press, Oxford.

Valdez, M.F. and C.R. Villalobos. - 1978. Distribución espacial, correlacion com el substrato y grado de agregación en Diadema antillarum Philippi (Echinodermata: Echinoidea). Ver. Biol. Trop., 26(1): 237-245.

Valentine, J.F., K.L. Heck Jr., J. Busby and D. Webb. - 1997. Experimental evidence that herbivory increases shoot density and productivity in a subtropical turtlegrass (Thalassia testudinum) meadow. Oecologia, 112: 193-200.

Weil, E., F. Losada and D. Bone. - 1984. Spatial variations in density and size of the echinoid Diadema antillarum Philippi on some Venezuelan coral reefs. Bijdragen tot de Dierkunde, 54(1): 73-82.

Wirtz, P. - 1995. Unterwasserführer Madeira, Kanaren and Azoren. Niedere Tiere, Delius Klasing, Edition Naglschmid, Stuttgart.

Wirtz, P. - 1998. Twelve invertebrate and eight fish species new to the marine fauna of Madeira, and a discussion of the zoogeography of the area. Helgoländer Meeresuntersuchungen, 52: 197-207.

Scient. ed.: J.M. Gili 